\title{
Laparoscopic diaphragmatic hernia repair using expanded polytetrafluoroethylene (ePTFE) for delayed traumatic diaphragmatic hernia
}

\author{
Yeseob Jee \\ Department of Surgery, Dankook University Hospital, Cheonan, South Korea
}

Videosurgery Miniinv 2017; 12 (2): 189-193

DOI: https://doi.org/10.5114/wiitm.2017.66373

\begin{abstract}
Traumatic diaphragmatic hernia (TDH) is an uncommon surgical problem, and diagnosis is often delayed. However, the mortality from bowel necrosis can reach $80 \%$. Therefore, suspicion is needed and surgery is required to prevent complications. A 50-year-old man was transferred due to abdominal pain and vomiting. Chest $X$-ray and computed tomography (CT) scan showed herniation of the stomach through the left diaphragm. The patient had fallen down 15 months ago and CT scan at that time revealed a small defect of the diaphragm without herniation. We diagnosed delayed herniation of TDH and the patient underwent laparoscopic repair using an expanded polytetrafluoroethylene (ePTFE) mesh. Recovery was uneventful and the CT scan at 3 months after the operation showed no recurrence. We reported a delayed presenting TDH and considered a laparoscopic approach to be safe and feasible during elective surgery. Moreover, use of an ePTFE mesh for repair of large diaphragmatic hernia was also feasible.
\end{abstract}

Key words: hernia, laparoscopic, diaphragm, expanded polytetrafluoroethylene.

\section{Introduction}

Traumatic diaphragmatic hernia is a rare surgical problem, and diagnosis is often delayed. At the time of the accident and in the emergency department, it is difficult to detect a small defect in the diaphragm using radiological findings, and related symptoms may not be recognized [1]. Therefore, diagnosis of traumatic herniation is frequently delayed until complications occur, e.g., cardiopulmonary compromise or gastrointestinal obstruction with or without strangulation. In addition, it is not uncommon to suspect that symptoms are associated with trauma when symptoms occur later [2, 3]. The clinical manifestation of diaphragmatic hernia differs depending on the side involved. Dyspnea and tachycardia are symptoms of right-side herniation, and gastrointestinal symptoms of left-side herniation. This is be- cause, in general, the herniated abdominal organs are the liver on the right side and the stomach, small intestine and large intestine on the left side [4].

In general, the treatment of diaphragmatic hernia has involved laparotomy or thoracotomy or both, since the first successful repair by Riolfi in 1886 [5]. Laparoscopic surgery is now widely accepted and laparoscopic diaphragmatic hernia repair is also performed. However, there is no study comparing open and laparoscopic surgery. Several methods can be used to repair defects, e.g., primary repair and repair with mesh. No study has reported an improved method of repairing diaphragmatic defects. Also, there is no study of the need for treatment of diaphragmatic injury without symptoms or hernia.

We reported successful treatment of a diaphragmatic hernia by laparoscopy using an expanded 

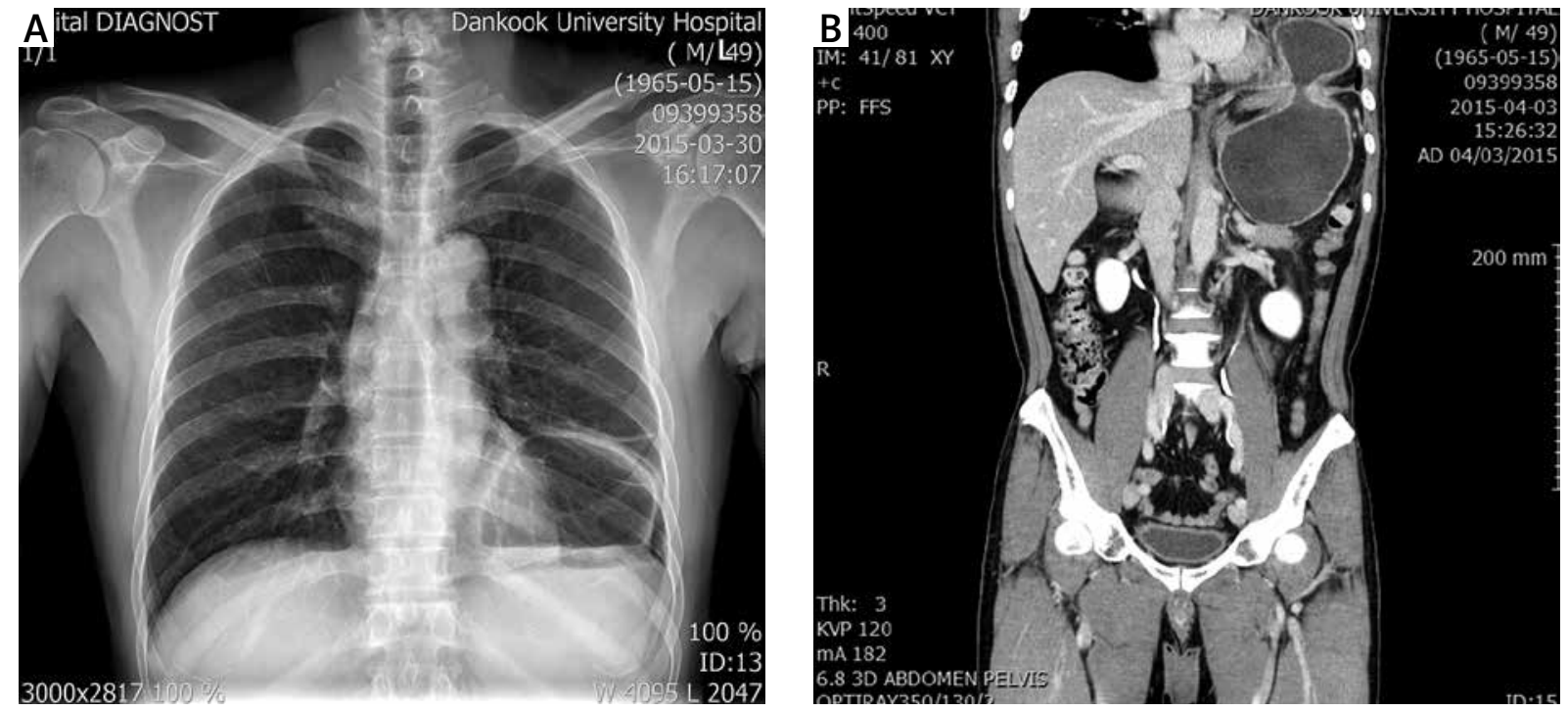

Photo 1. A - Chest X-ray showing left diaphragmatic hernia with bowel gas and subsegmental atelectasis on left lower lung field. B - Computed tomography (CT) scan of abdomen and pelvis showing herniation of gastric body and antrum through left diaphragm with organo-axial volvulus and obstruction at the pylorus level

polytetrafluoroethylene (ePTFE) mesh, together with a literature review.

\section{Case report}

A 50-year-old man was transferred to our emergency department from a local orthopedic clinic due

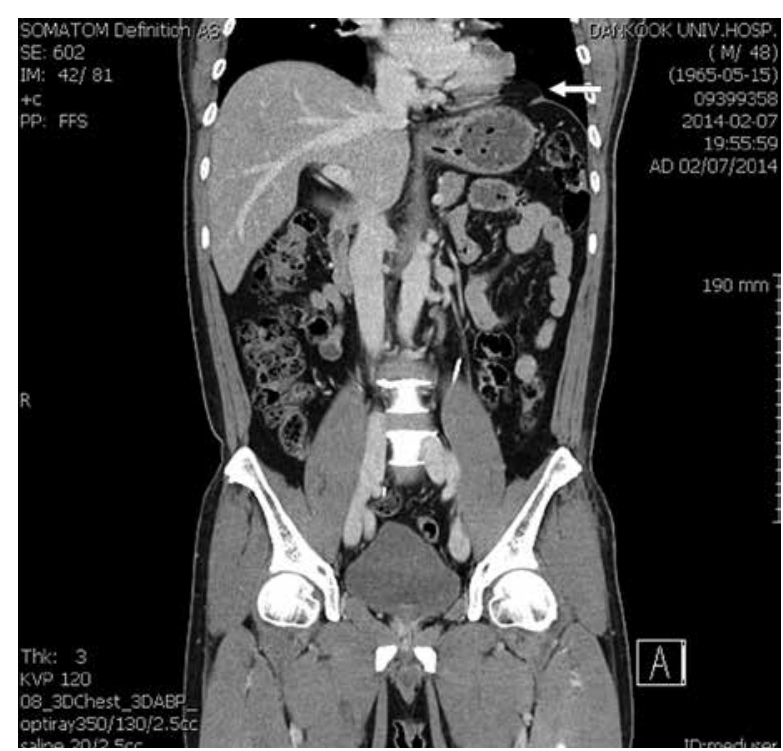

Photo 2. Computed tomography scan before 15 months showing small defect of left diaphragm (arrow) without herniation which we missed at that time to abdominal pain and vomiting for 6 days. The patient had been admitted to a local clinic for 6 days following surgery for fracture of his wrist joint. Epigastric pain developed and the patient underwent esophagoduodenoscopy, which led to a diagnosis of acute peptic ulcer. Use of ulcer medication, including a proton pump inhibitor, did not result in symptom improvement, and so the patient was transferred. His vital signs were stable and he showed mild epigastric tenderness on physical examination. Laboratory parameters revealed electrolyte imbalance and dehydration with a potassium level of $2.9 \mathrm{mmol} / \mathrm{l}$, chloride level of $87 \mathrm{mmol} / \mathrm{l}$, blood urea nitrogen level of $46.9 \mathrm{mg} / \mathrm{dl}$ and creatinine level of $1.40 \mathrm{mg} / \mathrm{dl}$. Chest X-ray (Photo $1 \mathrm{~A}$ ) and computed tomography (CT) scan (Photo $1 \mathrm{~B}$ ) showed herniation of the gastric body and antrum through the left diaphragm with organo-axial volvulus and obstruction at the pylorus level. To identify the cause of herniation, we rechecked his medical history and found that he had fallen a height of $5 \mathrm{~m} 15$ months prior. The CT scan at that time (Photo 2 ) revealed a small defect of the left diaphragm without herniation, which we had missed. The patient's symptoms improved after insertion of a nasogastric tube and hydration. The patient then underwent a laparoscopic examination (Photo 3). The patient was placed supine in the reverse Trendelenburg position with the surgeon on the right side of the patient. A total of 4 ports 

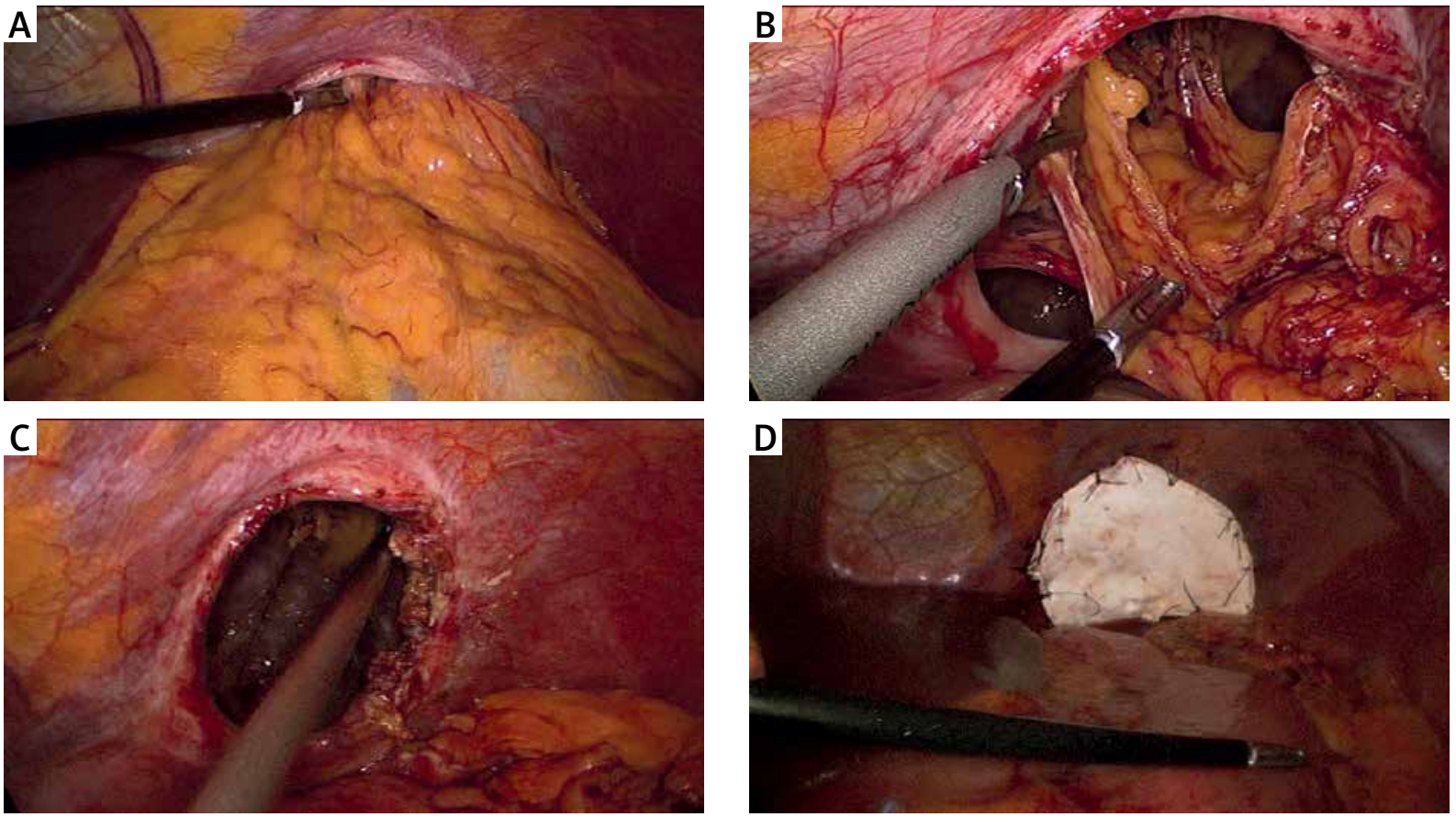

Photo 3. Laparoscopic view during operation. A - Herniation of stomach and omentum through defect of diaphragm. B - Dissection of omentum from diaphragm. C - About $6 \times 5 \mathrm{~cm}$ sized defect after completion of reduction. D - Repair of hernia with ePTFE mesh

were used: one $10 \mathrm{~mm}$ port at the umbilicus for the 30-degree rigid scope, one $12 \mathrm{~mm}$ and one $5 \mathrm{~mm}$ port at the right midcostal for working port and one $5 \mathrm{~mm}$ port at the left anterior axillary line for liver retraction. The gastric body and omentum were herniated through the defect in the left diaphragm. We reduced the herniated omentum and stomach using ultrasonic shear because a part of the omentum was attached to the diaphragm. After reduction, the defect in the diaphragm was about $6 \times 5 \mathrm{~cm}$ in size and the rim of the defect was firm and fibrous. Therefore, we considered primary repair to be impossible and decided to perform the repair using a mesh. Hence we cut ePTFE more than $2-3 \mathrm{~cm}$ beyond the defect area, patched an ePTFE mesh on the defect and fixed it 18 times with an interrupted prolene 3-O suture. Recovery was uneventful and the patient was discharged at 5 days after the operation. The patient underwent a follow-up CT scan at 3 months after the operation, and there was no recurrence or symptoms related to the operation (Photo 4).

\section{Discussion}

The incidence of diaphragmatic rupture after thoraco-abdominal trauma is $0.8-5 \%$, and more than
$30 \%$ of diaphragmatic hernia cases present late. The time of late presentation varied from $24 \mathrm{~h}$ to 50 years after trauma [4]. Grimes described the three phases of rupture of the diaphragm [6]. The acute phase is at the time of the injury to the diaphragm. The delayed phase is associated with transient herniation of the viscera, thus accounting for absent or intermittent non-specific symptoms. The obstruction phase involves complications of long-standing herniation, manifesting as obstruction, strangulation and rupture. The diaphragm is a septum that separates the abdominal cavity and the thoracic cavity. In normal patients, negative pressure was maintained in the thoracic cavity, and positive pressure in the abdominal cavity. This is important physiology of the abdominal cavity, thoracic cavity and diaphragm in terms of development of diaphragmatic hernia from small defects of the diaphragm. The negative pressure within the thoracic cavity then draws intra-abdominal contents into the chest for a prolonged period, even through small defects [7]. Therefore, if a diaphragmatic defect after trauma is detected on radiologic findings in the absence of hernia, surgery is mandatory regardless of symptoms. In this case, the defect in the diaphragm was small, and there 

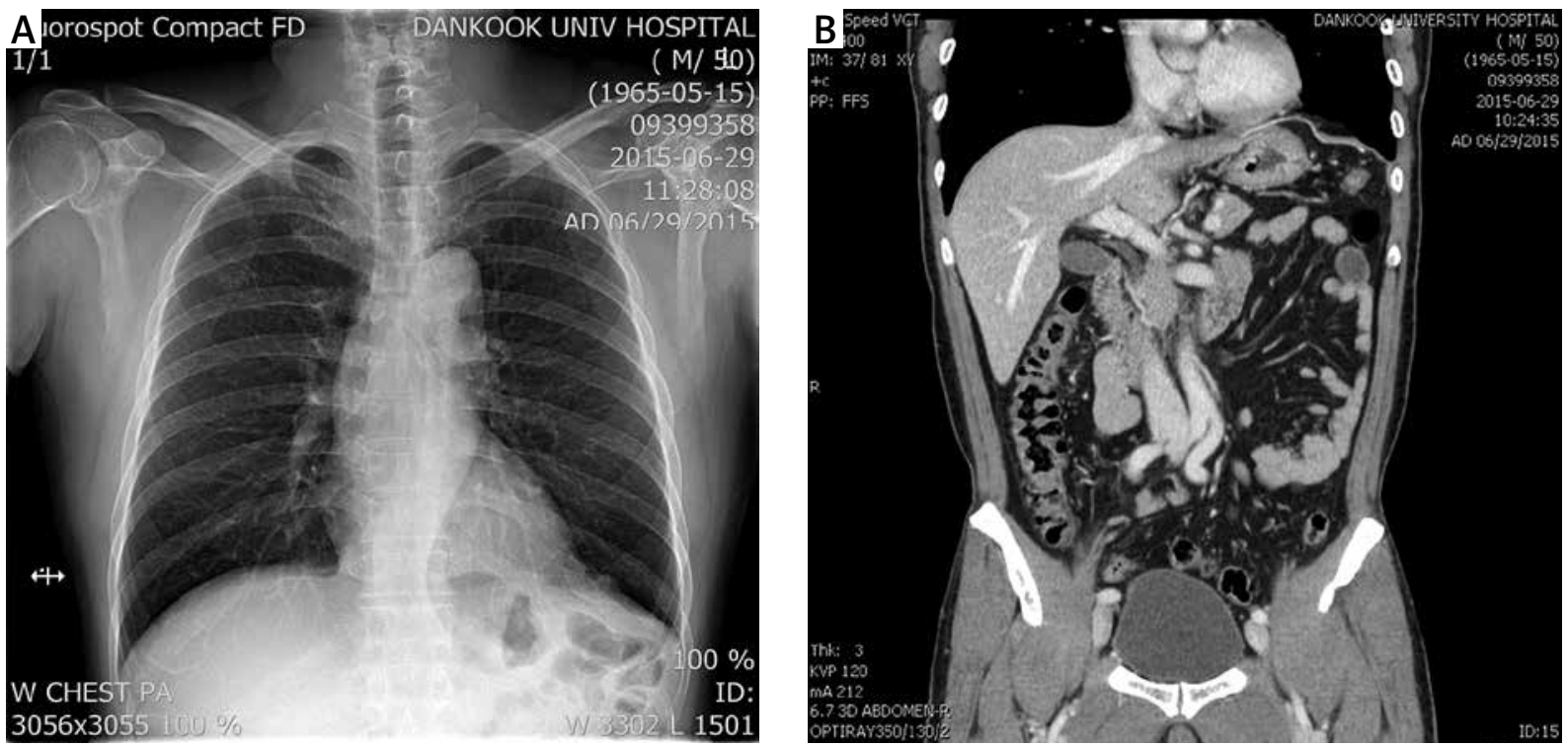

Photo 4. Chest PA (A) and CT scan (B) at 3 months after operation. There is no recurrence after the operation

was no herniation of abdominal organs. Diagnosis was missed at the time of trauma (Photo 2). However, the patient developed a large diaphragmatic hernia with gastric volvulus at 15 months after trauma. In addition, at the time of occurrence of abdominal pain, endoscopy did not confirm gastric volvulus. Perhaps it is because the endoscopist did not doubt the volvulus caused by diaphragmatic hernia. Thus, it is not easy to diagnose diaphragmatic hernia without suspicion.

The usual method for repair of diaphragmatic hernia is reduction of abdominal organs into the abdominal cavity through the diaphragmatic defect and repair of the diaphragm. The approach to reduction and repair of diaphragmatic hernia varies, and determining the optimum method is difficult. Thoracic surgeons tend to prefer a thoracic approach and general surgeons an abdominal approach. Therefore, abdominal, thoracic and both approaches were possible depending on the experience of the surgeon. However, the abdominal approach is mandatory in cases of suspicion or presence of bowel necrosis because of the inability to identify and repair bowel injury using a thoracic approach. Also, a laparoscopic or thoracoscopic approach can be used. The laparoscopic approach has been reported to be easier than the thoracoscopic approach. With the laparoscopic approach, manipulation of instruments and the bowel is easier than with thoracoscopy because of expansion of the abdominal cavity due to pneumoperitoneum [4, 8, 9]. The type of mesh used for repair of a diaphragmatic defect is controversial. Some authors have reported the use of prosthetic material as reinforcement, whereas other authors preferred simple suturing of the defect. However, it is generally agreed that defects larger than $20-30 \mathrm{~cm}^{2}$ require a prosthesis. Various synthetic mesh products are available [8]. We used an ePTFE mesh (Photo $3 \mathrm{D}$ ) because other types of mesh such as polypropylene induce excessive bowel adhesion and unacceptable rates of enterocutaneous fistula formation when placed intraperitoneally [10]. Furthermore, ePTFE differs from other synthetic mesh materials in that it is flexible and smooth. Therefore, it is easy to handle during repair and fixation of diaphragmatic defects.

The mortality rate from elective repair is low, but that from ischemic bowel secondary to strangulation can reach $80 \%$ [7]. Therefore, to prevent late, serious complications of diaphragmatic hernia, a high index of suspicion is needed and surgery is mandatory for late-presentation traumatic diaphragmatic hernia [4].

\section{Conclusions}

A laparoscopic approach was safe and feasible during elective surgery, and the use of an ePTFE synthetic mesh enabled repair of the large diaphragmatic hernia. 


\section{Conflict of interest}

The authors declare no conflict of interest.

\section{References}

1. Bani Hani MN. A combined laparoscopic and endoscopic approach to acute gastric volvulus associated with traumatic diaphragmatic hernia. Surg Laparosc Endosc Percutan Tech 2008; 18: 151-4.

2. Carter BN, Giuseffi J, Felson B. Traumatic diaphragmatic hernia. Am J Roentgenol Radium Ther 1951; 65: 56-72.

3. Dwari AK, Mandal A, Das SK, Sakar S. Delayed presentation of traumatic diaphragmatic rupture with herniation of the left kidney and bowel loops. Case Rep Pulmonol 2013; 2013: 814632.

4. Rashid F, Chakrabarty MM, Singh R, Iftikhar SY. A review on delayed presentation of diaphragmatic rupture. World J Emerg Surg 2009; 4: 32

5. Goh BK, Wong AS, Tay KH, Hoe MN. Delayed presentation of a patient with ruptured diaphragm complicated by gastric incarceration and perforation after appearently minor blunt trau ma. Can J Emerg Med 2004; 6: 277-80.

6. Grimes OF. Traumatic injuries of the diaphragm. Diaphragmatic hernia. Am J Surg 1974; 128: 175-81.

7. Wardi G, Lasoff D, Cobb A, Hayden S. Traumatic diaphragmtic hernia. J Emerg Med 2014; 46: 80-2.

8. Palnivelu C, Rangarajan M, Rajapandian S, et al. Laparoscopic repair of adult diaphragmatic hernias and eventration with primary sutured closure and prosthetic reinforcement: a retrospective study. Surg Endosc 2009; 23: 978-85.

9. Mccutcheon BL, Chin UY, Hogan GJ, et al. Laparoscopic repair of traumatic intrapericardial diaphragmatic hernia. Hernia 2010; 14: 647-9.

10. Townsend CM, Beauchamp RD Jr, Evers BM, Mattox KL. Sabiston textbook of surgery: the biological basis of modern surgical practice. $18^{\text {th }}$ ed. W.B.. Saunders, Philadelphia 2008; 1174

Received: 14.12.2016, accepted: 9.02.2017 